\title{
During the Initiation of Fermentation Overexpression of Hexokinase PII in Yeast Transiently Causes a Similar Deregulation of Glycolysis as Deletion of Tps1
}

\author{
JOSÉ ROBERTO ERNANDES $\dagger$, CATHERINE DE MEIRSMAN, FILIP ROLLAND, \\ JORIS WINDERICKX, JOHANNES DE WINDE, ROGELIO LOPES BRANDÃO† AND \\ JOHAN M. THEVELEIN* \\ Laboratorium voor Moleculaire Celbiologie, Katholieke Universiteit Leuven, Kardinaal Mercierlaan 92, \\ B-3001 Leuven-Heverlee, Flanders, Belgium
}

Received 18 June 1997; accepted 10 September 1997

In the yeast Saccharomyces cerevisiae a novel control exerted by $T P S 1 \quad(=G G S 1=F D P 1=B Y P 1=C I F 1$ $=G L C 6=T S S 1)$-encoded trehalose-6-phosphate synthase, is essential for restriction of glucose influx into glycolysis apparently by inhibiting hexokinase activity in vivo. We show that up to 50 -fold overexpression of hexokinase does not noticeably affect growth on glucose or fructose in wild-type cells. However, it causes higher levels of glucose-6-phosphate, fructose-6-phosphate and also faster accumulation of fructose-1,6-bisphosphate during the initiation of fermentation. The levels of ATP and Pi correlated inversely with the higher sugar phosphate levels. In the first minutes after glucose addition, the metabolite pattern observed was intermediate between those of the tps $1 \Delta$ mutant and the wild-type strain. Apparently, during the start-up of fermentation hexokinase is more rate-limiting in the first section of glycolysis than phosphofructokinase. We have developed a method to measure the free intracellular glucose level which is based on the simultaneous addition of D-glucose and an equal concentration of radiolabelled L-glucose. Since the latter is not transported, the free intracellular glucose level can be calculated as the difference between the total D-glucose measured (intracellular+periplasmic/extracellular) and the total L-glucose measured (periplasmic/extracellular). The intracellular glucose level rose in 5 min after addition of $100 \mathrm{~mm}$-glucose to $0 \cdot 5-2 \mathrm{~mm}$ in the wild-type strain, $\pm 10 \mathrm{~mm}$ in a $h x k 1 \Delta h x k 2 \Delta$ glkl $1 \Delta$ and $2-3 \mathrm{~mm}$ in a tps $1 \Delta$ strain. In the strains overexpressing hexokinase PII the level of free intracellular glucose was not reduced. Overexpression of hexokinase PII never produced a strong effect on the rate of ethanol production and glucose consumption. Our results show that overexpression of hexokinase does not cause the same phenotype as deletion of Tps1. However, it mimics it transiently during the initiation of fermentation. Afterwards, the Tps1-dependent control system is apparently able to restrict properly up to 50-fold higher hexokinase activity. (C) 1998 John Wiley \& Sons, Ltd.

Yeast 14: 255-269, 1998.

KEY WORDS - hexokinase PII; glycolysis; Tps1; fermentation

$\dagger$ Permanent address: Department of Biochemistry, Institute of Chemistry, São Paulo State University, C.P. 355, 14800-900 Araraquara, SP, Brazil.

$\$$ Permanent address: Laboratório de Bioquimica e Fisiologia de Microrganismos, Escola de Farmácia, Universidade Federal de Ouro Preto, Rua Costa Sena 171, 35.400.00 Ouro Preto, MG, Brazil.

*Correspondence to: J. M. Thevelein, Laboratorium voor Moleculaire Celbiologie, Katholieke Universiteit Leuven, Kardinaal, Mercierlaan 92, B-3001 Leuven-Heverlee, Flanders, Belgium. Tel: (+32) 16 321507; fax (+32) 16 321979; e-mail: johan.thevelein@bio.kuleuven.ac.be
Contract/grant sponsor: Belgian National Fund for Scientific Research.

Contract/grant sponsor: Belgian National Lottery.

Contract/grant sponsor: Research Fund of the Katholieke Universiteit Leuven.

Contract/grant sponsor: European Commission.

Contract/grant number: CHRX-CT93-0265.

Contract/grant number: ERBCI1*CT940101.

Contract/grant sponsor: Belgian Office for Cooperation with Developing Countries. 


\section{INTRODUCTION}

In the yeast Saccharomyces cerevisiae glucose is phosphorylated by hexokinase PI (encoded by $H X K 1$ ), hexokinase PII (encoded by $H X K 2$ ) and glucokinase (encoded by GLKI) (Entian, 1997). Recently a novel control apparently restricting hexokinase activity in vivo has been discovered. Yeast mutants which are deficient in the TPSI $(=G G S 1=F D P 1=B Y P 1=C I F 1=G L C 6=T S S 1)$ gene display a phenotype which is consistent with overactive hexokinase activity in vivo. The TPSI gene encodes trehalose-6-phosphate synthase, which catalyses the first reaction of trehalose biosynthesis (Bell et al., 1992; Vuorio et al., 1993). Tps1 mutants hyperaccumulate sugar phosphates and concomitantly deplete their ATP and free phosphate level, and in the long term also other phosphate-containing compounds, including the whole stock of polyphosphate (van de Poll et al., 1974; Van Aelst et al., 1991, 1993; Gonzalez et al., 1992). This metabolic deregulation has always been considered to be the cause of the inability of such strains to grow on rapidly-fermented sugars, like glucose and fructose. Recent work, however, has shown that other mechanisms appear to be involved in the specific growth defect on rapidlyfermented sugars in addition to the metabolic deregulation (Hohmann et al., 1996). Both the growth defect and the metabolic deregulation with glucose as carbon source are suppressed by a deletion of the $H X K 2$ gene, which encodes the most active sugar kinase isoenzyme, hexokinase PII (Hohmann et al., 1993). This result is consistent with unbridled hexokinase activity being the (main) cause of the metabolic deregulation and resulting growth defect of tpsl mutants. It supports the conclusion that in wild-type strains hexokinase activity is lower in vivo than would be anticipated from the activity measured in vitro and that this restricted activity is essential for the proper functioning of glycolysis.

Three different models have been proposed for the mechanism by which the TPSI gene product might restrict glucose influx into glycolysis by inhibiting hexokinase activity (for a recent discussion, see Thevelein and Hohmann, 1995). The first model proposes that the Tps1 protein has an additional function to its trehalose-6-phosphate synthase activity and that this function is responsible for triggering interaction between glucose transporters and hexokinase resulting in inhibition of hexokinase activity (Thevelein, 1992). The second model is based on the observation that stimulation of glycerol production also suppresses the growth defect on glucose of tps1 mutants, albeit only after a long lag phase. It proposes that hexokinase activity itself is not restricted in vivo and that the excess of sugar phosphate formed is channelled into trehalose synthesis in order to recover the free phosphate, which in turn is required in glycolysis at the level of glyceraldehyde-3-phosphate dehydrogenase (Hohmann et al., 1993). The third model is based on the finding that trehalose-6-phosphate acts in vitro as a competitive inhibitor (with respect to glucose) of both hexokinase isoenzymes and could therefore directly inhibit hexokinase activity in vivo (Blazquez et al., 1993). Recent work using a strain with both a reduced level of Tps1 protein and an enhanced level of trehalose-6-phosphate compared to the wild-type strain has made the second model unlikely, at least as the main cause of the growth defect (Hohmann et al., 1996). Although part of the results obtained with this strain are consistent with a role of trehalose-6-phosphate in restricting hexokinase activity in vivo, other results indicate involvement of additional mechanisms, such as those proposed in the first model (Hohmann et al., 1996).

In the present work, we have investigated whether strong overexpression of hexokinase activity would cause the same growth defect on glucose and fructose, as observed in the tpsl $\Delta$ strain. In addition, we have tried to obtain evidence that hexokinase activity itself is truly inhibited in vivo by measuring glucose-induced accumulation of sugar phosphates in strains overexpressing hexokinase PII, the most active isoenzyme. The results indicate that in such strains the pattern of glycolytic metabolites observed in the first minutes after addition of glucose indeed approaches the pattern of tpsl mutants. This is consistent with the presence of highly elevated hexokinase activity in vivo in the latter strains. Unexpectedly, in the strains overexpressing hexokinase, the accumulation of fructose-1,6biphosphate, the product of the subsequent phosphofructokinase reaction, was also clearly stimulated.

The rate by which yeast cells ferment glucose to ethanol is generally thought to be controlled mainly by the activity of glucose transport, phosphofructokinase, pyruvate kinase and the rate of ATP consumption. Although there is no agreement in the literature on the extent by which these 
steps each contribute to flux control in glycolysis, there appears to be a general agreement that the contribution of the other glycolytic enzymes is negligible (Gancedo and Serrano, 1989; Galazzo and Bailey, 1990). In previous work no effect of overexpression of glycolytic enzymes, including hexokinase, on the total glycolytic flux under steady-state conditions could be detected (Heinisch, 1986; Schaaff et al., 1989). Davies and Brindle (1992) showed that overexpression of phosphofructokinase increased glycolytic flux but only under aerobic conditions and apparently due to a compensatory decrease in the flux in oxidative phosphorylation. No significant changes in glycolytic intermediate levels were detected. As opposed to these previous studies in which only steady-state conditions were used, we have measured in yeast strains with strong overexpression of hexokinase the accumulation of intracellular sugar phosphates and of ethanol in the medium as a function of time during the initiation of fermentation with glucose. Our results indicate that hexokinase is a ratelimiting factor for the glycolytic flux during the initiation of fermentation.

\section{EXPERIMENTAL PROCEDURES}

\section{Yeast strains and overexpression constructs}

The following isogenic strains have been used: W303-1A (wild type; MATa leu2-3/112 ura3-1 trp1-1 his3-11/15 ade2-1 can1-100 GAL SUC2; Thomas and Rothstein, 1989), YSH290 (MATa leu2-3/112 ura3-1 trp1-1 his3-11/15 ade2-1 can1-100 GAL SUC2 tps1::TRP1) and YSH7.4-3C (MATa leu2-3/112 ura3-1 trp1-1 his3-11/15 ade2-1 can1-100 GAL SUC2 hxk1::HIS3 hxk2::LEU2 glk1 ::LEU2). For overexpression of hexokinase PII, the wild-type strain was transformed with a multi-copy vector (YEplac195+URA3) carrying either the $H X K 2$ gene behind its own promoter (YEp- $H X K 2$ ) or behind the strong constitutive $P G K$ promoter (YEp- $P G K / H X K 2$ ). For the latter, the coding region of $H X K 2$ was amplified by PCR using primers that introduced a unique restriction site upstream of the start codon and downstream of the stop codon. The amplification product was cloned into a vector (YEplac- $P G K$ ) containing the $P G K$ promoter and the $P G K$ transcription termination sequences. The construct was completely checked by dideoxysequencing to confirm that the PCR amplification did not introduce sequencing errors in the coding region of $H X K 2$. The cells were transformed using the lithium acetate protocol (Ito et al., 1983) as modified by Eberhardt and Hohmann (1995).

\section{Growth and incubation conditions}

The strains overexpressing hexokinase were pregrown overnight in synthetic medium with $4 \%$ galactose and lacking uracil. For the other strains the medium was supplemented with uracil $(30 \mathrm{mg} / \mathrm{ml})$. The cultures were grown in an orbital shaker $(200 \mathrm{rpm})$ at $30^{\circ} \mathrm{C}$ and harvested by centrifugation at the early stationary phase. They were resuspended in complete YP medium $(2 \%$ peptone, $1 \%$ yeast extract) supplemented with $2 \%$ ethanol and allowed to grow for a further $3 \mathrm{~h}$. The cells were then harvested by centrifugation, cooled in ice and washed twice with cold YP medium.

\section{Sampling conditions and determination of metabolites}

For measurement of glycolytic intermediates, the cells were resuspended in YP medium or 2-(Nmorpholino)ethanesulphonic acid (Mes) buffer and incubated in a reciprocating water bath shaker at $30^{\circ} \mathrm{C}$. After $15 \mathrm{~min}$ of shaking, glucose was added to obtain a specific final concentration (20, $50,100$ or $250 \mathrm{~mm})$. Samples of $3 \mathrm{ml}$ containing approximately $150 \mathrm{mg}$ of cells (wet weight) were withdrawn at the indicated times and immediately sprayed in methanol medium at $-40^{\circ} \mathrm{C}$ as described by de Koning and van Dam (1992). The cells were washed with the same methanol medium at $-40^{\circ} \mathrm{C}$. Each time great care was taken to remove the supernatant completely by aspiration after any remaining liquid had been allowed to drain from the sides of the tubes by standing in the bath at $-40^{\circ} \mathrm{C}$. This reduced as much as possible carry over of glucose from the medium. Intracellular metabolites were subsequently extracted according to the protocol of de Koning and van Dam (1992). Their levels were determined essentially as described by Bergmeyer (1974). The protein concentration was quantified according to Lowry et al. (1951).

\section{Determination of free intracellular glucose}

For determination of free intracellular glucose an equal amount of $100 \mathrm{~mm}$-D-glucose and $100 \mathrm{~mm}^{14} \mathrm{C}$-radiolabelled L-glucose was added to the cells (total radioactivity of $10 \times 10^{6} \mathrm{cpm}$ in a volume of $33.75 \mathrm{ml}$ ) and the cells were 
incubated and extracted as described for glycolytic metabolites. The total amount of D-glucose associated with the cells (intracellular+ periplasmic/extracellular) was measured with the classical hexokinase-based method. The total amount of L-glucose associated with the cells (periplasmic/extracellular) was determined by counting the radioactivity present in the extract. The free intracellular D-glucose level was determined by subtracting the level of L-glucose from the total level of D-glucose.

\section{Preparation of crude enzyme extracts and determination of enzyme activities}

Samples containing approximately $50 \mathrm{mg}$ cells (wet weight) were withdrawn and collected by centrifugation for $3 \mathrm{~min}$ at $4^{\circ} \mathrm{C}$. The pellet was washed twice with $5 \mathrm{ml}$ imidazole buffer $\mathrm{pH} 7 \cdot 0$. The cells were broken by vigorous mixing for $2 \mathrm{~min}$ in a small precooled test tube containing $1 \mathrm{~g}$ of glass beads $(0.5 \mathrm{~mm}$ diameter) and resuspended in $2 \mathrm{ml}$ of the imidazole buffer containing $1 \mathrm{~mm}$ PMSF. The crude enzyme extract was centrifuged for $5 \mathrm{~min}$ at $13000 \mathrm{rpm}$ and $4^{\circ} \mathrm{C}$. Hexokinase activity was measured according to Maitra and Lobo (1971). For the enzyme assays protein was determined using the microbiuret method (Zamenhoff, 1957).

\section{Determination of glucose consumption and ethanol production}

For determination of ethanol production, the cells were harvested and washed twice with Mes buffer ( $25 \mathrm{~mm}, \mathrm{pH} 5 \cdot 5-6 \cdot 0)$. Cells (1 g wet weight) were suspended in either $44 \mathrm{ml} \mathrm{YP}$ medium or Mes buffer and incubated at $30^{\circ} \mathrm{C}$ with shaking. After 30 min pre-incubation, glucose was added in the desired concentration $(5,10,20,50,100$ and $250 \mathrm{~mm})$. Samples were withdrawn by filtration at different times and the glucose consumed and ethanol produced determined in the filtrate. Ethanol was determined enzymatically with a commercial kit (Boehringer Mannheim). Glucose was determined with the glucose oxidase-peroxidase method.

\section{Reproducibility of the results}

The experiments were repeated at least three times with consistent results. Representative results are shown.
Table 1. Hexokinase activity as measured in cell extracts with either glucose or fructose as substrate (mU/mg protein).

\begin{tabular}{lrr}
\hline & Glucose & Fructose \\
\hline Wild type & 201 & 282 \\
Wild type +YEp- $H X K 2$ & 4250 & 4040 \\
Wild type +YEp- $P G K / H X K 2$ & 13230 & 10850 \\
$t p s 1 \Delta$ & 213 & 268 \\
\hline
\end{tabular}

Values are means of three independent experiments. The cells were grown in the same way as for the metabolite measurements (see Materials and Methods).

\section{RESULTS}

Growth on glucose and accumulation of glycolytic metabolites in hexokinase-overexpressing strains

We have compared in this work the capacity to grow on glucose and the effect of glucose addition on the accumulation of glycolytic metabolites in four strains: the wild-type strain, the tps $1 \Delta$ strain and two strains overexpressing hexokinase. One of the latter was a wild-type strain transformed with a multi-copy plasmid carrying the $H X K 2$ gene behind its own promoter, while the other strain contained the same plasmid but with the $H X K 2$ gene behind the strong, constitutive $P G K$ promoter (for details on these constructs, see Materials and Methods). Hexokinase activity as measured in cell extracts was respectively about 20-fold (YEp-HXK2) and about 50-fold (YEp$P G K / H X K 2)$ elevated compared to the wild-type strain (Table 1). None of the two strains with overexpression of hexokinase showed a detectable growth problem, neither on glucose- nor on fructose-containing medium (results not shown).

In the first set of experiments $20 \mathrm{~mm}$-glucose was added to cells growing in rich ethanol medium and the accumulation of sugar phosphates (glucose6-P, fructose-6-P and fructose-1,6-bisP) in the cells was determined together with the level of ATP and phosphate for the next $3 \mathrm{~min}$. Figure 1 shows that the glucose-6-P and fructose-6-P levels are consistently higher in the hexokinase overexpression strains compared to the wild-type strain but not as high as in the tpsl $\Delta$ strain. The glucose-6-P and fructose-6-P levels in the hexokinase overexpression strains still show the typical 'overshoot' observed in the wild-type strain. In the tps $1 \Delta$ strain the levels of the two sugar phosphates remain high, consistent with the idea that it lacks a control that 

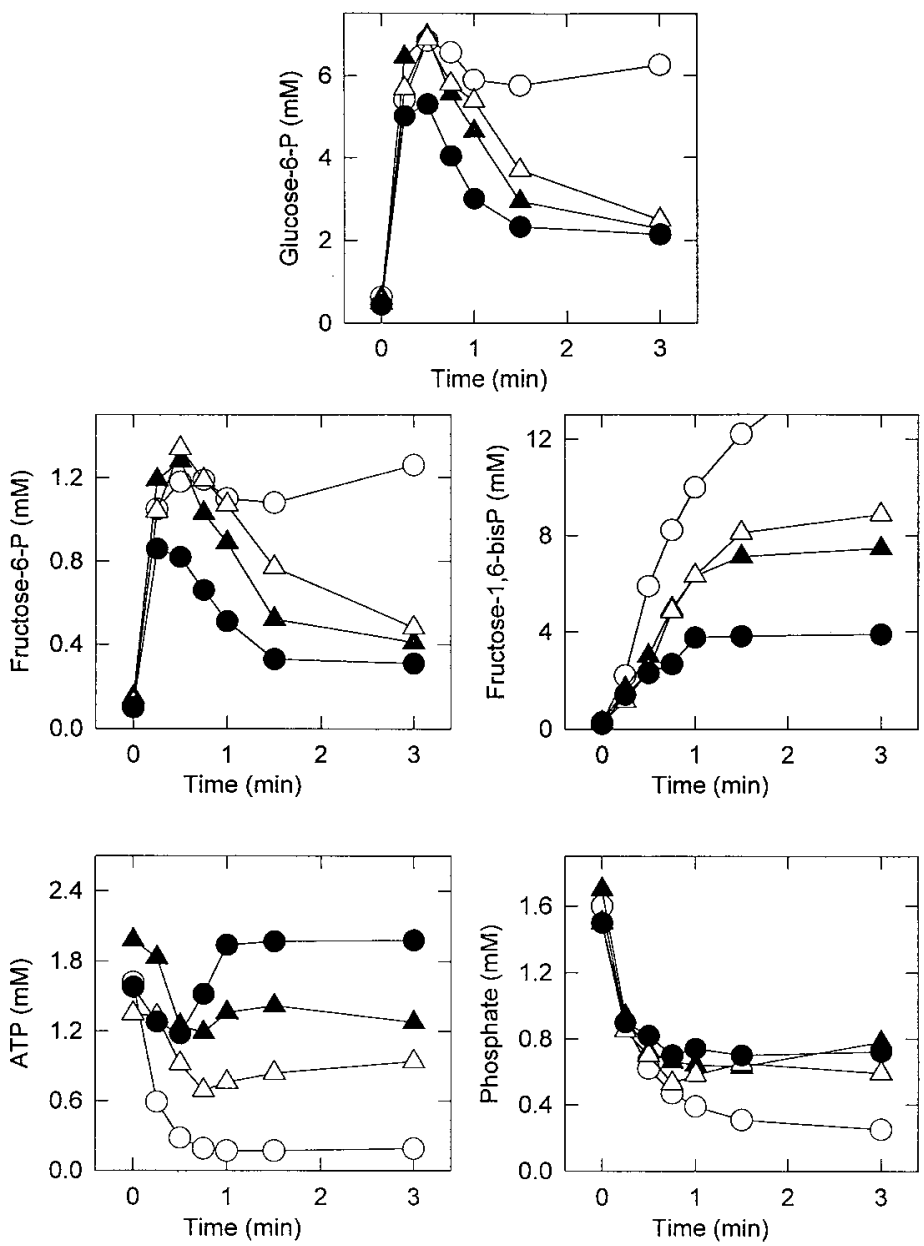

Figure 1. Levels of intracellular metabolites after addition of $20 \mathrm{~mm}$-glucose to YPethanol-growing cells of the wild-type strain $(\bullet)$, the tps $1 \Delta$ strain $(\bigcirc)$, the YEp- $H X K 2$ overexpression strain $(\boldsymbol{\Delta})$ and the YEp- $P G K / H X K 2$ overexpression strain $(\triangle)$.

becomes operative shortly after the initiation of glucose metabolism, as opposed to the three other strains. These results show that the novel system restricting glucose influx into glycolysis, which is deficient in the tps $1 \Delta$ strain, has the capacity to properly downregulate an excess of at least 50 times the normal hexokinase activity in a wild-type strain. The level of fructose-1,6-bisP in the hexokinase overexpression strains was also clearly elevated compared to the level in the wild-type strain apparently indicating that in the first minutes after addition of glucose the activity of 6-phosphofructo-1-kinase is limited by the availability of its substrate fructose-6-P. In the tps $1 \Delta$ strain, fructose-1,6-bisP accumulation was still much higher, which is consistent with this conclusion. In all cases the effect with the strain overexpressing the $H X K 2$ gene from the $P G K$ promoter was more pronounced than with $H X K 2$ behind its own promoter, which fits with the enzymatic activity as determined in vitro (Table 1). This further confirms that the changes observed in the metabolite levels in vivo are truly due to the enhanced activity of hexokinase.

The changes in the accumulation pattern of the sugar phosphates compared to the pattern in the wild-type strain were inversely correlated with the course of the ATP level and, somewhat less pronounced, the level of free phosphate (Figure 1). This excludes that changes in the protein cell 
volume ratio are responsible for the elevated levels of the sugar phosphates. In the wild-type strain, the level of ATP transiently dropped just after addition of glucose. The initial drop in the ATP level correlated with the initial sharp increase in the glucose-6-P and fructose-6-P level, and is consistent with very high (unrestricted) hexokinase activity in the first $30 \mathrm{~s}$ after addition of glucose. After this time, the Tps1-dependent control apparently starts to inhibit hexokinase activity, reducing the rate of glucose phosphorylation and therefore, following this interpretation, allowing the ATP level to recover due to ATP synthesis in the lower part of glycolysis. In the hexokinase overexpression strains, the Tps1-dependent inhibition system needs more time to restrict glucose influx, as shown by the slower return of the sugar-6-P levels after the initial increase. As a result, the ATP level also needs more time for recovery. On the other hand, in the tpsls strain, no restriction on glucose influx is imposed and the ATP level is unable to recover at all. The phosphate level in a wild-type strain typically drops with about $50 \%$ in the first minutes after addition of glucose. In the strains overexpressing hexokinase the drop is slightly more pronounced while in the tps $1 \Delta$ strain it is clearly more pronounced (Figure 1).

The results obtained with an extracellular glucose concentration of $20 \mathrm{~mm}$ were confirmed in experiments in which concentrations of $50 \mathrm{~mm}$ and $100 \mathrm{~mm}$ glucose were used and in which the metabolites were measured also over a longer time period $(30 \mathrm{~min})$. In this respect it is important to realize that several glucose carriers with different $K_{\mathrm{m}}$ values are responsible for glucose transport in yeast cells. This is also true for derepressed cells which display a major high-affinity component in glucose transport. Some of the glucose carriers have $K_{\mathrm{m}}$ values as high as $50-100 \mathrm{~mm}$ (Reifenberger et al., 1997). In the experiment where $50 \mathrm{~mm}$-glucose was added, the effect of hexokinase overexpression on the glycolytic metabolites was more pronounced. The progressive drop in the free phosphate level with increasing hexokinase activity was now also very clear (Figure 2). This confirms that glucose transport was not yet saturated. In the experiments with $100 \mathrm{~mm}$ extracellular glucose the metabolite pattern during the first $3 \mathrm{~min}$ in the strains overexpressing hexokinase now resembled even more the pattern in the tps $1 \Delta$ strain (Figure 3). Apparently, they experience a similar but transient metabolic deregulation. This was clearly manifested in the ATP level which dropped initially as in the tpsl $\Delta$ strain and recovered only slowly afterwards. Subsequently, the strains overexpressing hexokinase recovered from the transient deregulation of glycolysis, as opposed to the tps $1 \Delta$ strain. Their metabolite profile started to deviate from that of the tpsl $1 \Delta$ strain after about 1-2 min depending on the metabolite and started to resemble more the pattern in the wild-type strain. After $30 \mathrm{~min}$, however, in both strains overexpressing hexokinase, the profile of some metabolites (fructose-1,6-bisP and ATP) was still clearly different from the wild-type profile. Other metabolites (glucose-6-P, fructose-6-P and $\mathrm{Pi}$ ) had nearly recovered to the wild-type level. Also the pyruvate level in the strains overexpressing hexo-kinase was intermediate between the level in the tps $1 \Delta$ strain and the wild-type strain (results not shown).

The same experiments were also performed with cells suspended in Mes ( $\mathrm{pH}$ 6) buffer to which $50 \mathrm{~mm}$ - and $250 \mathrm{~mm}$-glucose (for $60 \mathrm{~min}$ ) were added (results not shown). Similar results were obtained as in rich medium except that the differences between the wild-type strain and the strains overexpressing hexokinase were smaller at the later time points. The initial deregulation of metabolism, however, was very similar to the one observed in rich medium, and as a result the ATP level dropped in the two strains overexpressing hexokinase nearly as much as in the tpsl $\Delta$ strain. In the latter strain the ATP level did not recover, whereas in the other strains it recovered with a rate again inversely correlated with the degree of hexokinase overexpression.

\section{Determination of the free intracellular glucose level}

We have developed a method to measure precisely the free intracellular glucose level. It is based on the absence of significant transport of L-glucose by the yeast glucose carriers (Heredia et al., 1968; Gamo et al., 1995). Control experiments confirmed that addition of $500 \mathrm{~mm}-\mathrm{L}$-glucose together with 100 mm-D-glucose had no significant effect on the accumulation of intracellular glucose metabolites (results not shown). The total glucose level associated with the cells after harvesting, washing and extraction with the cold methanol $\left(-40^{\circ} \mathrm{C}\right)$ quenching method (de Koning and van Dam, 1992) is apparently composed of several fractions: 

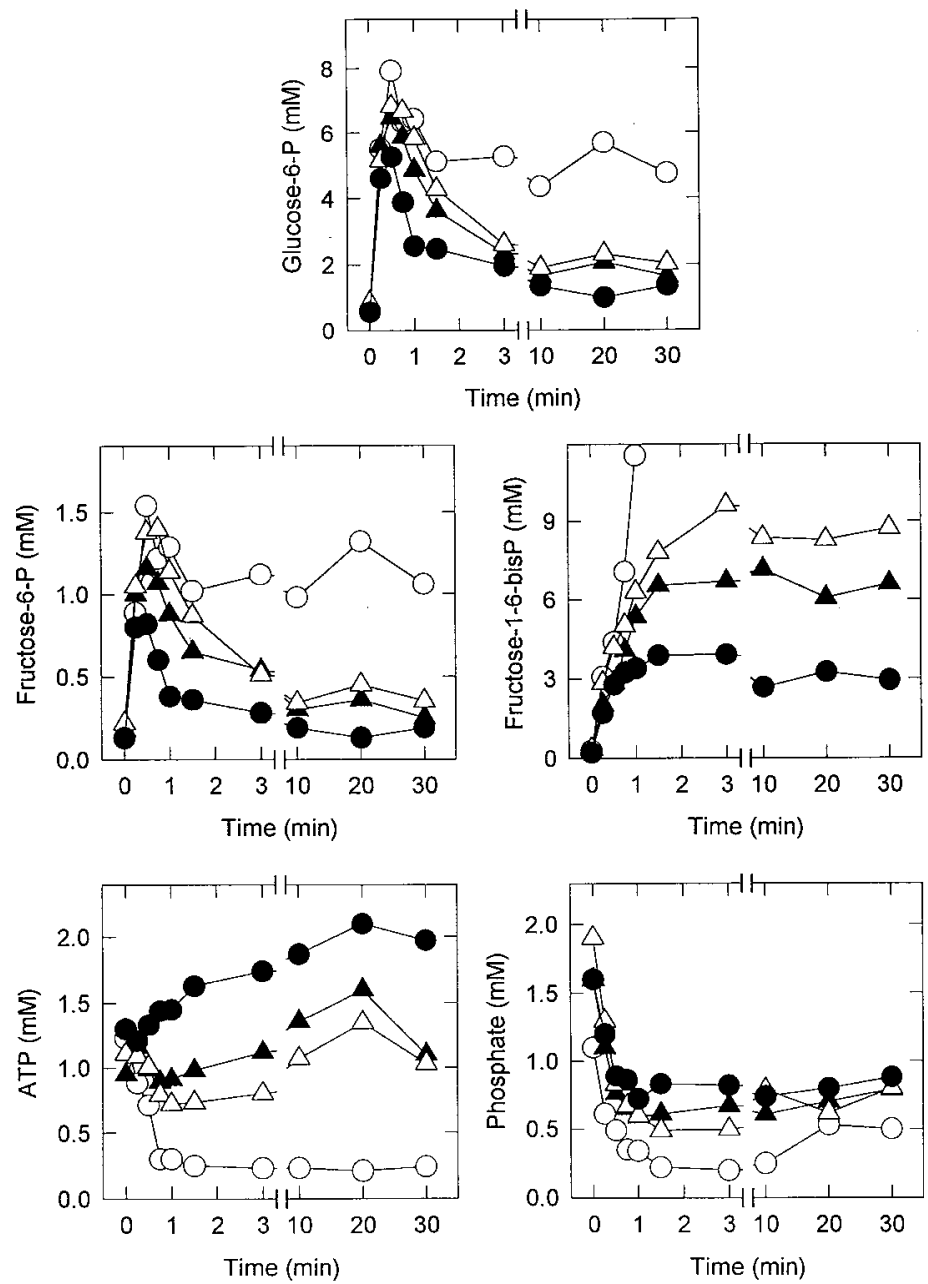

Figure 2. Levels of intracellular metabolites after addition of $50 \mathrm{~mm}$-glucose to YPethanol-growing cells of the wild-type strain $(\bullet)$, the $t p s 1 \Delta$ strain $(\bigcirc)$, the YEp- $H X K 2$ overexpression strain $(\mathbf{\Lambda})$ and the YEp- $P G K / H X K 2$ overexpression strain $(\triangle)$.

glucose located truly intracellularly (cytosolic glucose), glucose located in the periplasm/cell wall and possibly glucose that freezes to the exterior of the cells and cannot be washed away with cold methanol $\left(-40^{\circ} \mathrm{C}\right)$. To correct for the latter two fractions we have added an equal amount of ${ }^{14} \mathrm{C}$-radioactively labelled $\mathrm{L}$-glucose to the cells together with the D-glucose. Since L-glucose is not taken up to a significant extent by the glucose transport system, it can only be located in the periplasm/cell wall and extracellular fractions. The amount of L-glucose present can be determined easily from the radioactivity measured. Because equal amounts of D- and L-glucose were added, the periplasm/cell wall and extracellular fractions should contain the same level of both. The total amount of D-glucose is determined with the standard glucose-oxidase peroxidase method. The level of free intracellular D-glucose can then be determined by subtracting the level of L-glucose from the total level of D-glucose measured. The free intracellular D-glucose level determined in this way should still be considered approximate because it is determined by subtraction of two values which are both relatively large compared to the free D-glucose level.

For wild-type cells this method indicated values of $0.5-2 \mathrm{~mm}$ for the free intracellular D-glucose 

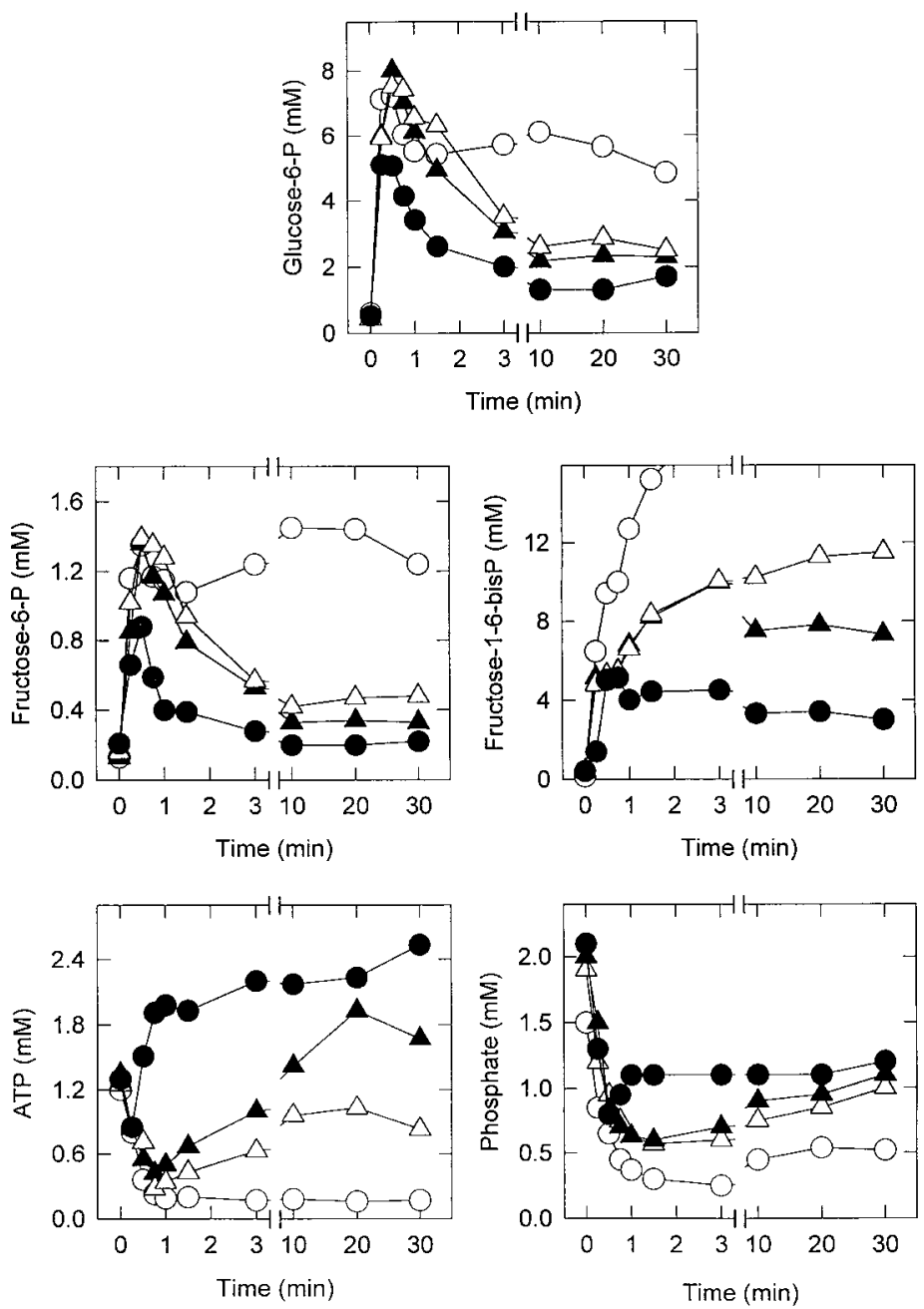

Figure 3. Levels of intracellular metabolites after addition of $100 \mathrm{~mm}$ -

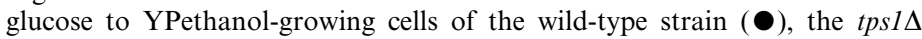
strain $(\bigcirc)$, the YEp- $H X K 2$ overexpression strain $(\mathbf{\Delta})$ and the YEp-PGK/ $H X K 2$ overexpression strain $(\triangle)$.

level (Figure 4). A control experiment with a triple

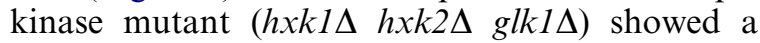
rapid biphasic increase in the free glucose level up to about $10 \mathrm{~mm}$ in $5 \mathrm{~min}$. The biphasic increase probably reflects the contribution from different glucose carriers: after the rapid initial increase to a few $\mathrm{mm}$ the high-affinity glucose carriers cease to mediate netto-transport because of their $K_{\mathrm{m}}$ of 1-2 mM (Walsh et al., 1994). Other carriers with a higher $K_{\mathrm{m}}$ (Reifenberger et al., 1997) then mediate the continued but slower increase in the free glucose level. In the tps $1 \Delta$ strain the free glucose level was somewhat elevated
(2-3 mM) compared to the wild-type strain (Figure 4), in spite of its phenotype apparently indicating higher hexokinase activity in vivo. In the strains with overexpression of hexokinase, the intracellular free glucose level was certainly not reduced compared to the wild-type strain (Figure 4).

Ethanol production and glucose consumption in hexokinase-overexpressing strains

We also measured in the four strains the accumulation of ethanol from glucose with a range of 
A.
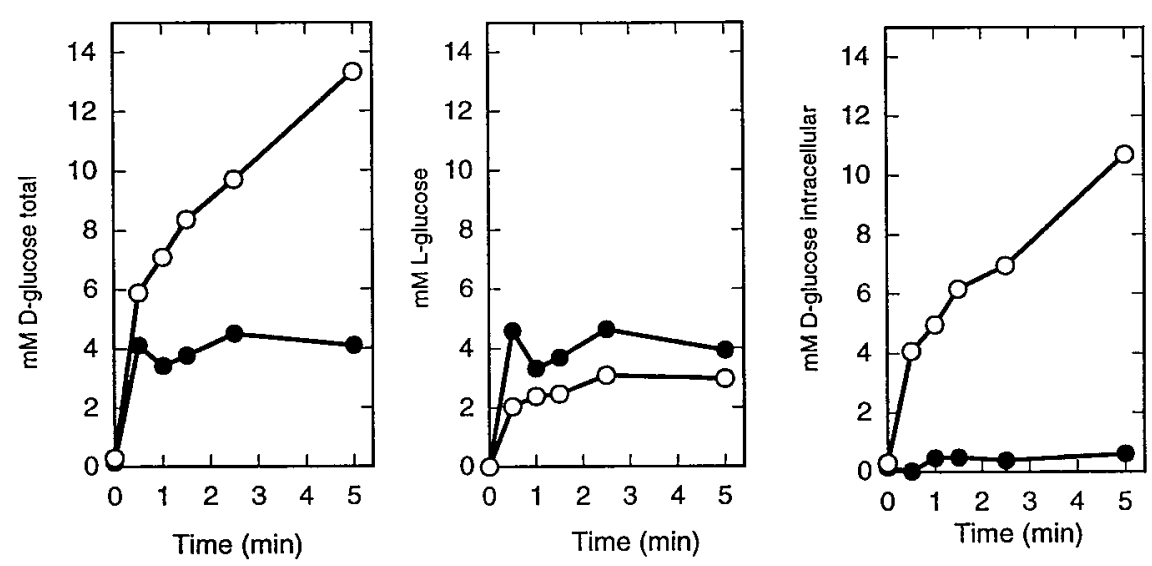

B.
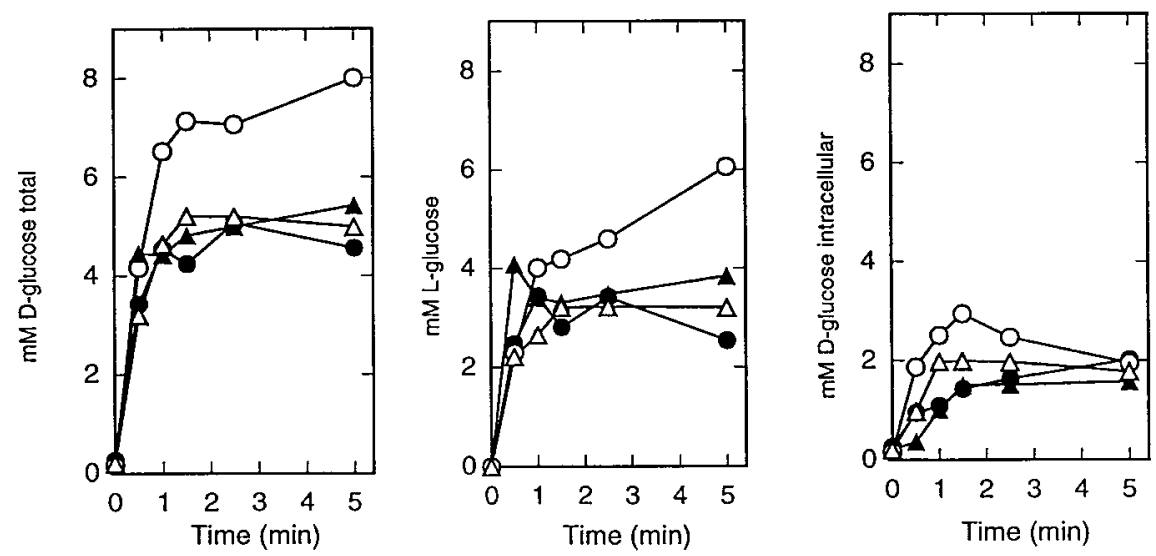

Figure 4. Levels of free intracellular D-glucose, as determined from the total level of D-glucose (intracellular+periplasmic/extracellular) minus the level of L-glucose (periplasmic/extracellular). (A) Wild-type strain $(\bullet), h x k 1 \Delta h x k 2 \Delta$ glkl $1 \Delta$ strain $(\bigcirc)$. (B) Wild-type strain $(\bullet), t p s 1 \Delta$ strain $(\bigcirc)$, YEp- $H X K 2$ overexpression strain $(\mathbf{\Lambda})$ and the YEp- $P G K / H X K 2$ overexpression strain $(\triangle)$.

glucose concentrations $(5,10,20,50,100$ and $250 \mathrm{~mm})$ and both in rich medium and in Mes buffer. In the same cultures glucose consumption was also measured. The higher the external glucose concentration, the lower the relative amount of ethanol produced and the amount of glucose consumed by the tpsl $1 \Delta$ strain compared to the other strains. This fits with previous results showing that tpsl mutants are more sensitive to high glucose concentrations than to low glucose concentrations (Neves et al., 1995). In rich medium the tpsl $\Delta$ strain started to deviate from the other strains already at the lowest external glucose concentrations $(5-10 \mathrm{~mm})$ whereas in Mes buffer this occurred at higher concentrations (20-50 mM) (results not shown). In general the strains overexpressing hexokinase did not show a higher rate of ethanol production and glucose consumption than the wild-type strain. Only in Mes buffer at the lowest external glucose concentrations a slight increase was observed (Figure 5). At higher glucose concentrations ( $>50 \mathrm{~mm}$ ) in Mes buffer the difference became insignificant (not shown). In rich medium there was only a slight difference at the lowest glucose concentrations (5-10 mM) (Figure 5). In Mes buffer and in the presence of a glucose concentration of $10 \mathrm{~mm}$ the tps $1 \Delta$ strain also showed somewhat faster ethanol production and glucose consumption than the wild-type strain (Figure 5). 

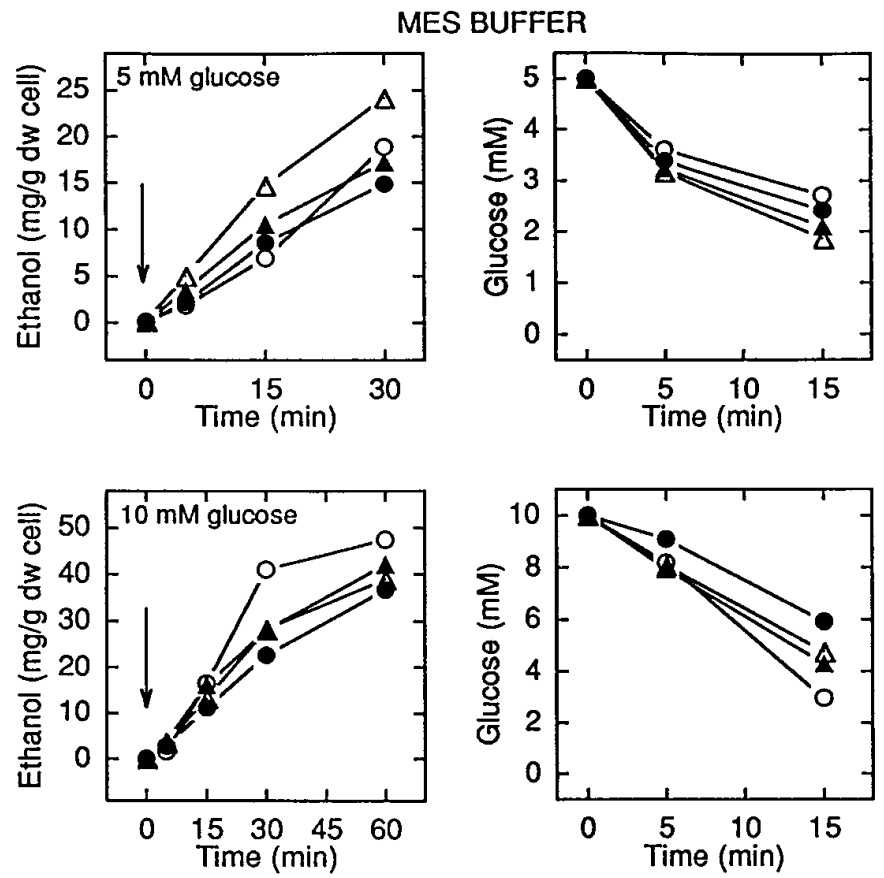

YP MEDIUM
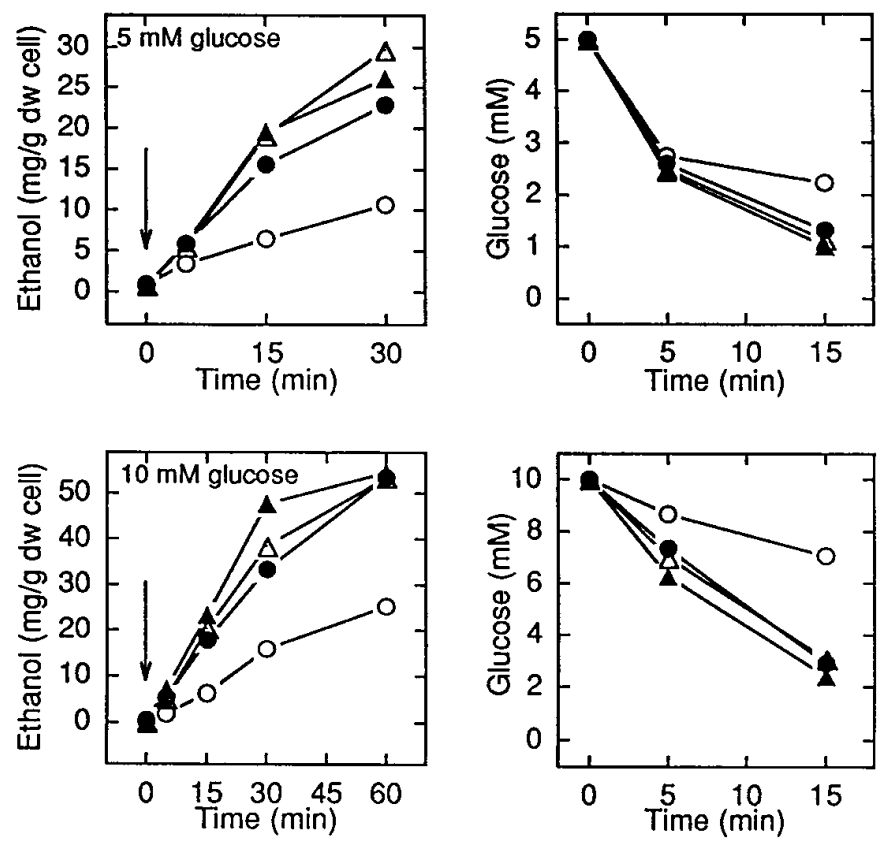

Figure 5. Ethanol production and glucose consumption in Mes buffer and YP medium with $5 \mathrm{~mm}$ - and $10 \mathrm{~mm}$-glucose. Wild-type strain (O), tps1 $1 \Delta$ strain $(\bigcirc)$, YEp- $H X K 2$ overexpression strain $(\boldsymbol{\Delta})$ and YEp$P G K / H X K 2$ overexpression strain $(\triangle)$. 


\section{DISCUSSION}

The recent discovery of the Tps1-dependent control on glucose influx into glycolysis, apparently by inhibition of hexokinase activity, has raised the question as to the effect of hexokinase overexpression on the capacity to grow on glucose, the pattern of glycolytic metabolites and the flux through glycolysis. In previous work with a strain containing a multi-copy plasmid with the $H X K 2$ gene, no adverse effects on the capacity to grow on glucose were reported and no effect on the rate of ethanol formation under steady-state conditions was observed (Schaaff et al., 1989). In addition to a similar strain with a multi-copy plasmid containing the $H X K 2$ gene, we have also used a strain with the $H X K 2$ gene behind the $P G K$ promoter that showed about 50 -fold higher hexokinase activity as measured in vitro. Since none of the two strains showed a growth problem neither with glucose nor with fructose as carbon source, the Tps1dependent control is apparently able to cope with a very large excess of hexokinase activity. This has important consequences for any model trying to explain how the trehalose-6-phosphate synthase curtails hexokinase activity. The absence of a negative effect of overexpression of hexokinase in wild-type cells is not due to glucose transport being or becoming rate-limiting, since deletion of the TPSI gene results in excessive accumulation of sugar phosphates and a growth problem on glucose. When glucose carrier genes are deleted, a tps $1 \Delta$ mutant becomes less sensitive to glucose, confirming that reduction of glucose supply can limit hexokinase activity in vivo to such an extent that the Tps1 control becomes less important (Neves et al., 1995). Overexpression of hexokinase in a $\operatorname{tps} 1 \Delta$ strain further aggravates the growth problem (as shown by the near absence in such strains of spontaneous suppressor mutants that grow again on glucose; unpublished results). This indicates that even in a tps $1 \Delta$ mutant, glucose transport has not become limiting for hexokinase activity in vivo.

On the other hand, precise determination of the glycolytic metabolites just after addition of glucose shows that hexokinase overexpression does have a clear effect on the glycolytic metabolite pattern during the initiation of fermentation. It mimics to some extent the effect of deletion of TPS1. The overshoot in the levels of glucose-6-P and fructose6-P are accentuated by hexokinase overexpression and the same is true for the transient drop in the
ATP level and the drop in free phosphate. However, the effects of hexokinase overexpression are only transient. After a few minutes already the Tps1-dependent control system is able to master the influx of glucose into glycolysis in these strains.

Interestingly, the accentuation in the hexokinase overexpression strains of the transient increase in sugar phosphate and transient drop in ATP gives the impression that also in wild-type cells glycolysis starts with uncontrolled hexokinase activity. If this is true it has the important consequence that the Tps1-dependent control system is not yet operative at the moment glucose is added. Initiation of glucose metabolism would trigger its formation and/or activation. Depending on the model that has been proposed for the mechanism of the Tps1-dependent control, this has the following consequences. In the model where an interaction is proposed between Tps1, hexokinase and sugar transport, it would mean that the interaction does not exist before glucose addition and that it is initiated only when for instance Tps1 and/or hexokinase become catalytically active. As a result of this, a possible interaction of these proteins would never be revealed in experiments measuring initial glucose transport (which is classically measured over a period of at most a few seconds). The mechanism of the second model carries an inherent delay for the action of the Tps1-dependent control since it is based on deviation of the surplus of glucose-6-P formed into trehalose synthesis. Since trehalose-6-P synthase is activated by fructose-6-P and inhibited by free phosphate (Vandercammen et al., 1989; Londesborough and Vuorio, 1991), the enzyme might become only fully active in vivo after the fructose-6-P level has increased and the phosphate level has dropped. Absence of the Tps1-dependent control at the moment of glucose addition has important consequences for the third model since in this model hexokinase activity is inhibited in vivo by trehalose-6-P and the trehalose-6-P concentration is the same before glucose addition and during exponential growth on glucose (Blazquez et al., 1993; Hohmann et al., 1996). Hence, if the Tps1-dependent control has to be installed during initial glucose metabolism, the control proposed by the third model would not only be absent before glucose addition but also during exponential growth on glucose. Interestingly, we have recently found that the trehalose-6phosphate concentration shows a dramatic, but transient increase just after addition of glucose (Hohmann et al., 1996). This seems to support that 
trehalose-6-phosphate inhibition of hexokinase, at least if it is relevant in vivo, is only installed during the initiation of glucose metabolism. However, at the same time it implies that trehalose-6-phosphate inhibition of hexokinase cannot be relevant during exponential growth on glucose since its level then is the same as before addition of glucose. During exponential growth on glucose other mechanisms would have to be involved in the inhibition of hexokinase. Hence, if the Tps1-dependent control were absent before glucose metabolism had been initiated this would have important consequences for the possible mechanism involved.

The recent discovery of the novel Tps1dependent control restricting glucose influx into glycolysis has raised new questions concerning the control of glycolytic flux. Previously, hexokinase has always been considered to have an insignificant contribution to the control of glycolytic flux (Gancedo and Serrano, 1989; Galazzo and Bailey, 1990). The activity of hexokinase in vivo was thought to be limited by substrate availability and hence by the previous glucose transport step. Our data show that overexpression of hexokinase in a wild-type strain clearly stimulates the accumulation of sugar phosphates after addition of glucose and therefore apparently also the flux in the first part of glycolysis (up to fructose-1,6-bisphosphate), at least during an initial time period after the addition of glucose. The changes in the level of the other relevant metabolites, such as ATP and phosphate, are in agreement with this conclusion, Hence, our results show that during the initiation of fermentation hexokinase activity is not simply limited by the glucose transport step and that it has an important contribution to the control of glycolytic flux during its initiation. On the other hand, under all conditions and at all time points the concentrations of glucose-6-P and fructose-6-P showed the same ratio. This seems to indicate that phosphoglucoisomerase has very little control over the flux during the start-up of glycolysis.

The level of fructose-1,6-bisP increased faster in the hexokinase overexpression strain compared to the wild-type strain. This could be due to higher phosphofructokinase activity or to lower activity of aldolase (or downstream enzymes). The second possibility might be caused by protein burden effects (Snoep et al., 1995) on the lower part of glycolysis. However, this seems less likely because the differences are already visible after $15 \mathrm{~s}$ and because there is no deleterious effect of hexokinase overexpression on ethanol production and glucose consumption. The rate of phosphofructokinase in vivo, as is true for any enzyme, can be limited in three ways: (1) its inherent maximal activity, (2) any control reducing its inherent maximal activity, and (3) its substrate supply. Since there is no evidence that overexpression of hexokinase would affect transcription/translation of phosphofructokinase so as to enhance its inherent activity or that it would affect a control limiting phosphofructokinase activity in vivo, we feel that our results indicate that during the initiation of glycolysis phosphofructokinase activity is most likely restricted by its substrate supply.

Apparently, the contribution of other glycolytic enzymes in the second part of glycolysis to the control of the overall glycolytic rate is large since under most conditions we could not observe an effect of hexokinase overexpression on the rate of ethanol production. Under some conditions, i.e. at low glucose concentrations and in Mes buffer, there was a slight stimulation of ethanol production in the strains overexpressing hexokinase. Possibly, such an effect might also be observed if glucose repression were installed faster in the strains overexpressing hexokinase. Hexokinase PII is known to be essential for glucose repression, but the exact role of the enzyme in this respect is unclear (Rose et al., 1991). On the other hand, the small stimulation of ethanol production was accompanied by a small increase in the glucose consumption rate, suggesting a true, albeit limited, increase in the overall glycolytic flux.

Determination of the free intracellular glucose level in yeast cells fermenting glucose has always been difficult with classical harvesting and extraction methods. There is possible carry over of glucose from the medium and washing of the cells, on the other hand, can easily reduce the intracellular glucose level. This cold-methanolquenching method developed by de Koning and van Dam (1992) should in principle allow us to circumvent this problem because it allows us to wash any extracellular glucose away at a temperature of $-40^{\circ} \mathrm{C}$ where metabolism of the intracellular glucose should be completely absent. However, it appears that a significant amount of glucose gets frozen in the periplasmic space and/or the cell wall and/or to the exterior of the cells. Even with repeated washings it is not possible to remove this glucose. To measure precisely the intracellular glucose level, we have taken advantage of the well-known observation that L-glucose is not transported to a significant extent by the yeast 
glucose transporters (Heredia et al., 1968; Gamo et al., 1995). Addition of a five-fold higher level of L-glucose together with D-glucose had no significant effect on the appearance of intracellular glucose metabolites, confirming that L-glucose does not inhibit the uptake of D-glucose. Simultaneous addition of $100 \mathrm{mM}-\mathrm{D}$-glucose and 100 mm-radioactively labelled L-glucose allowed an estimation of the intracellular level of D-glucose from the difference between the total amount of D-glucose, measured enzymatically, and the level of L-glucose, located extracytoplasmatically and determined on the basis of its specific radioactivity.

In the wild-type strains the free intracellular glucose level appears to be about $0 \cdot 5-2 \mathrm{~mm}$, which is higher than the $K_{\mathrm{m}}$ of the hexokinases $(0 \cdot 1-$ $0.2 \mathrm{~mm}$ ) and of glucokinase $(0.03 \mathrm{~mm})$ (Entian, 1997). Hence, this result fits with the conclusion in this paper that hexokinase activity, at least during the initiation of yeast fermentation, is not limited by substrate availability. The absence of a decrease in the free intracellular glucose level in the strains overexpressing hexokinase does not fit with the changes in the other metabolites. If hexokinase activity in vivo is enhanced, one would expect the free intracellular glucose level to rise more slowly during the start-up of glycolysis. We had already observed previously that the free glucose level in tps 1 mutants rose higher than in the wild-type strains (Hohmann et al., 1993; Van Aelst et al., 1993) and this is now confirmed with the new method (Figure 4). In principle this is also inconsistent with the higher hexokinase activity apparently present in vivo in such strains. In the tps $1 \Delta$ mutant the L-glucose level seemed to increase somewhat with time, which might be due to a permeability problem caused by the pleiotropic $\operatorname{tps} 1 \Delta$ mutation. If L-glucose had somehow leaked into the tps $1 \Delta$ cells, the calculated free intracellular D-glucose level should have been even higher than the level reported. The unexpected behaviour of the free intracellular glucose level might indicate the existence of novel, unknown controls operating between glycolysis and glucose transport. Alternatively overexpression of hexokinase PII might in some way alter the expression pattern of the glucose carriers with a different free intracellular glucose level as a result.

In conclusion, our results are consistent with the idea that in wild-type yeast cells incoming glucose is initially phosphorylated in an unrestricted way and that this causes a transient metabolic deregulation until the Tps1-dependent control sets in and limits in some way glucose phosphorylation. Overexpression of hexokinase clearly accentuates the temporary metabolic problem occurring immediately after addition of glucose. However, the Tps1dependent control is able to overcome rapidly even a 50-fold excess of hexokinase activity, allowing normal growth on glucose and fructose. The clear stimulating effect of hexokinase overexpression on the accumulation of fructose-1,6-bisP indicates that hexokinase has an important control on the flux in the first part of glycolysis during the start-up of fermentation. Finally, the unexpected behaviour of the intracellular free glucose level might indicate the existence of novel unknown interactions between glycolysis and glucose transport.

\section{ACKNOWLEDGEMENTS}

J.R.E. is a recipient of a visiting fellowship from Conselho Nacional de Desenvolvimento Cientifico e Tecnologico do Brazil (CNPqRHAE). C.D.M. acknowledges receipt of a post-doctoral fellowship from the Institute for Scientific and Technological Research (IWT, Brussels, Belgium), J.W. from the Belgian National Fund for Scientific Research (NFWO) and J.d.W. from the European Commission (Human Capital and Mobility Programme ERBCHBG-CT93-0284). This work was supported by grants from the Belgian National Fund for Scientific Research (FGWO), the Belgian National Lottery, the Research Fund of the Katholieke Universiteit Leuven, the European Commission (Human Capital and Mobility Programme CHRX-CT93-0265; International Scientific Cooperation ERBCI1*CT940101) and the Belgian Office for Cooperation with Developing Countries (ABOS).

\section{REFERENCES}

Bell, W., Klaasen, P., Ohnacker, M., et al. (1992). Characterization of the $56-\mathrm{kDa}$ subunit of yeast trehalose-6-phosphate synthase and cloning of its gene reveal its identity with the product of $C I F 1$, a regulator of carbon catabolite inactivation. Eur. J. Biochem. 209, 951-959.

Bergmeyer, H. U. (1974). Methods of Enzymatic Analysis, 2nd edn. Academic Press, New York.

Blazquez, M. A., Lagunas, R., Gancedo, C. and Gancedo, J. M. (1993). Trehalose-6-phosphate, a new regulator of yeast glycolysis that inhibits hexokinases. FEBS Lett. 329, 51-54. 
Davies, S. E. C. and Brindle, K. M. (1992). Effects of overexpression of phosphofructokinase on glycolysis in the yeast Saccharomyces cerevisiae. Biochemistry 31, 4729-4735.

de Koning, W. and van Dam, K. (1992). A method for the determination of changes of glycolytic metabolites in yeast on a subsecond time scale using extraction at neutral pH. Anal. Biochem. 204, 118-123.

Eberhardt, I. and Hohmann, S. (1995). Strategy for deletion of complete open reading frames in Saccharomyces cerevisiae. Curr. Genet. 27, 306-308.

Entian, K.-D. (1997). Sugar phosphorylation in yeast In Zimmerman, F. K. and Entian, K.-D. (Eds), Yeast Sugar Metabolism: Biochemistry, Genetics, Biotechnology and Applications. Technomic Publishing Co. Lancaster, Pennsylvania, U.S.A. pp. 67-79.

Galazzo, J. L. and Bailey, J. E. (1990). Fermentation pathway kinetics and metabolic flux control in suspended and immobilized Saccharomyces cerevisiae. Enzym. Microb. Technol. 12, 162-172.

Gamo, F. J., Moreno, E. and Lagunas, R. (1995). The low-affinity component of the glucose transport system in Saccharomyces cerevisiae is not due to passive diffusion. Yeast 11, 1393-1398.

Gancedo, C and Serrano, R (1989) Energy-yielding metabolism. In Rose, A. H. and Harrison, J. S. (Eds), The Yeasts 2nd edn, vol. 3. Academic Press Inc., London, New York, pp. 205-259.

Gonzalez, M. I., Stucka, R., Blazquez, M. A., Feldmann, H. and Gancedo, C. (1992). Molecular cloning of $C I F 1$, a yeast gene necessary for growth on glucose. Yeast 8, 183-192.

Heinisch, J. (1986). Isolation and characterization of the two structural genes coding for phosphofructokinase in yeast. Mol. Gen. Genet. 202, 75-82.

Heredia, C. F., Sols, A. and de la Fuente, G. (1968). Specificity of the constitutive hexose transport in yeast. Eur. J. Biochem. 5, 321-329.

Hohmann, S., Bell, W., Neves, M. J., Valckx, D. and Thevelein, J. M. (1996). Evidence for trehalose-6phosphate-dependent and -independent mechanisms in the control of sugar influx into yeast glycolysis. Mol. Microbiol. 20, 981-991.

Hohmann, S., Neves, M. J., de Koning, W., Alijo, R., Ramos, J. and Thevelein, J. M. (1993). The growth and signalling defects of the $g g s 1$ ( $f d p 1 / b y p l)$ deletion mutant on glucose are suppressed by a deletion of the gene encoding hexokinase PII. Curr. Genet. 23, 281-289.

Ito, H., Fukuda, Y., Murata, K. and Kimura, A. A. (1983). Transformation of intact yeast cells treated with alkali cations. J. Bacteriol. 153, 163-168.

Londesborough, J. and Vuorio, O. (1991). Trehalose6-phosphate synthase/phosphatase complex from bakers' yeast: purification of a proteolytically activated form. J. Gen. Microbiol. 137, 323-330.

Lowry, O. H., Rosebrough, N. J., Farr, A. L. and Randall, R. J. (1951). Protein measurement with the Folin phenol reagent. J. Biol. Chem. 193, 265-275.

Maitra, P. K. and Lobo, Z. (1971). Control of glycolytic enzyme synthesis in yeast by products of the hexokinase reaction. J. Biol. Chem. 246, 489-499.

Neves, M. J., Hohmann, S., Bell, W., et al. (1995). Control of glucose influx into glycolysis and pleiotropic effects studied in different isogenic sets of Saccharomyces cerevisiae mutants in trehalose biosynthesis. Curr. Genet. 27, 110-122.

Reifenberger, E., Boles, E. and Ciriacy, M. (1997). Kinetic characterization of individual hexose transporters of Saccharomyces cerevisiae and their relation to the triggering mechanisms of glucose repression. Eur. J. Biochem. 245, 324-333.

Rose, M., Albig, W. and Entian, K.-D. (1991). Glucose repression in Saccharomyces cerevisiae is directly associated with hexose phosphorylation by hexokinase PI and hexokinase-PII. Eur. J. Biochem. 199, 511-518.

Schaaff, I., Heinisch, J. and Zimmermann, F. K. (1989). Overproduction of glycolytic enzymes in yeast. Yeast 5, 285-290.

Snoep, J. L., Yomano, L. P., Westerhoff, H. V. and Ingram, L. O. (1995). Protein burden in Zymomonas mobilis: negative flux and growth control due to overproduction of glycolytic enzymes. Microbiol. 141, 2329-2337.

Thevelein, J. M. (1992). The RAS-adenylate cyclase pathway and cell cycle control in Saccharomyces cerevisiae. Antonie van Leeuwenhoek, Journal of Microbiology. Special Issue: 'Molecular Biology of Yeasts' (Grivell, L. (Ed.), Kluwer, Dordrecht) 62, 109-130.

Thevelein, J. M. and Hohmann, S. (1995). Trehalose synthase: guard to the gate of glycolysis in yeast? Trends Biochem. Sci. 20, 3-10.

Thomas, B. J. and Rothstein, R. (1989). Elevated recombination rates in transcriptionally active DNA. Cell 56, 619-630.

Van Aelst, L., Hohmann, S., Zimmermann, F. K., Jans, A. W. H. and Thevelein, J. M. (1991). A yeast homologue of the bovine lens fibre MIP gene family complements the growth defect of a Saccharomyces cerevisiae mutant on fermentable sugars but not its defect in glucose-induced RAS-mediated cAMP signalling. EMBO J. 10, 2095-2104.

Van Aelst, L., Hohmann, S., Bulaya, B., et al. (1993). Molecular cloning of a gene involved in glucose sensing in the yeast Saccharomyces cerevisiae. Mol. Microbiol. 8, 927-943.

van de Poll, K. W., Kerkenaar, A. and Schamhart, D. H. J. (1974). Isolation of a regulatory mutant of fructose-1,6-diphosphatase in Saccharomyces carlsbergensis. J. Bacteriol. 117, 965-970.

Vandercammen A., François, J. and Hers, H.-G. (1989). Characterization of trehalose-6-phosphate synthetase 
and trehalose-6-phosphate phosphatase of Saccharomyces cerevisiae. Eur. J. Biochem. 182, 613-620.

Vuorio, O. E., Kalkkinnen, N. and Londesborough, J. (1993). Cloning of two related genes encoding the $56-\mathrm{kDa}$ and $123-\mathrm{kDa}$ subunits of trehalose synthase from the yeast Saccharomyces cerevisiae. Eur. J. Biochem. 216, 849-861.
Walsh, M. C., Smits, H. P., Scholte, M. and van Dam, K. (1994). Affinity of glucose transport in Saccharomyces cerevisiae is modulated during growth on glucose. J. Bacteriol. 176, 953-958.

Zamenhoff, S. (1957). Preparation and assay of deoxyribonucleic acids from animal tissues. Methods Enzymol. 3, 696-704. 